\title{
EDOUARD VAILLANT
}

A son retour d'exil en 1880 , Vaillant ne voit, dans un avenir immédiat, qu'une tâche principale: l'organisation d'un grand parti socialiste national, se donnant pour but premier la conquête du pouvoir politique. Nominalement lié à l'organisation blanquiste issue de la proscription londonienne, le Comité révolutionnaire central, ${ }^{1}$ il s'établit loin de Paris pour se vouer à l'organisation du parti dans son département du Cher; c'est le premier témoignage de son désaccord avec les représentants d'idées, de tactiques devenues à ses yeux surannées: c'étaient celles du Second Empire. Pour cette tâche d'organisation il a craint d'abord de s'allier avec Jules Guesde, le passé de celui-ci étant trop équivoque. ${ }^{2}$ Mais Vaillant se rend compte bientôt qu'entre Guesde et lui existe une communauté certaine d'esprit touchant cette œuvre immédiate d'organisation; dans les années quatre-vingts, les rapports entre les deux hommes se resserrent jusqu'à atteindre, au moment de la crise boulangiste, le niveau d'une véritable unité d'action. Au cours des années quatre-vingt-dix, après le départ du C.R.C. des éléments entraînés dans l'aventure boulangiste par Henri Rochefort, Vaillant dirige son parti vers une organisation ouverte et active sur une triple base: action politique sous toutes ses formes, y compris l'usage de toutes les fonctions électives; ${ }^{3}$ action économique de toute espèce; action démocratique, création d'un mouvement de masse entraînant, dans le courant socialiste, les forces vives de la nation. ${ }^{4}$ Poursuivant constamment cette forme d'action il s'éloigne des «doctrinaires» du

1 Pendant les années quatre-vingts, bien que Vaillant donne son adhésion au C.R.C., ses désaccords avec les blanquistes restaient nombreux et profonds. Cf. $\mathrm{J}$. Howorth, Edouard Vaillant and the unification of the French socialist party (Thèse à paraître en 1972).

2 Lié aux bakouninistes en 1872, aux radicaux en 1876, Guesde vient de passer au marxisme.

3 Vaillant est conseiller municipal de Paris (1884-93) et député de la Seine (18931915).

4 En 1893 Vaillant lance une campagne en faveur de la démocratie directe. 
P.O.F. pour trouver, pendant quelques années, en Jaurès un compagnon de lutte avec lequel il s'est senti sûrement plus d'affinités. ${ }^{1} \mathrm{La}$ crise provoquée par l'affaire Dreyfus, puis le problème Millerand, le rejettent vers les Guesdistes, faisant revivre une ancienne entente, ambiguë peut-être, fondée en tout cas sur l'affirmation principale que le socialisme doit rester avant tout révolutionnaire. Jouant alors un rôle de trait d'union entre Jaurès et Guesde, soucieux d'éviter une scission, qui surviendra pourtant en 1901, il se voue entièrement à la cause de l'unité; son rôle ne sera pas des moindres quand il s'agira d'obliger Jaurès à choisir entre le socialisme révolutionnaire et le millerandisme réformiste. Vaillant est un des architectes principaux de l'unité de 1905, un but qu'il poursuit depuis quelques vingt ans. Après la consolidation de la Seconde Internationale par suite de la création du Bureau socialiste international en 1900 , Vaillant prête tout le poids de son autorité à la cause de la paix européenne. Le désespoir que provoque chez lui la faillite de l'Internationale en août 1914, son patriotisme, ravivé immédiatement par les souvenirs de 1870, une conviction solide que, des flammes de la guerre, surgira un ordre social nouveau, l'amènent à soutenir, jusqu'à sa mort en 1915, l'effort de guerre de la France. Quelques jours avant sa mort, il s'ouvrit à Louis Dubreuilh: "cette guerre m'a tué... avoir lutté 40 ans pour l'écarter, pour la conjurer et avoir été forcé de la subir, atroce, implacable! C'est l'écroulement de tout mon être.»²

Pour bien comprendre le développement de la pensée d'Edouard Vaillant après la Commune, il faut se rappeler ses années d'exil, années critiques où, s'éloignant d'une formation jusqu'alors plutôt éclectique, il se voit contraint de suivre un chemin plus étroit. A cette époque, bien qu'armé d'une connaissance toujours plus approfondie des théories marxistes, Vaillant, élu dès son arrivée à Londres membre du Conseil général de l'Internationale, s'éloigne progressivement, à partir de 1872 , de la coterie de Marx par suite d'un profond malentendu entre les deux hommes touchant la tactique à suivre immédiatement dans les divers pays européens. ${ }^{3}$ Il préfère allier son activité à la

1 Le lien entre matérialisme et idéalisme sur le plan philosophique, entre objectivisme et subjectivisme sur le plan politique, bien que conçu d'une façon différente par les deux hommes, explique en partie cette liaison assez précaire. 2 L. Dubreuilh, "A la mémoire d'Edouard Vaillant", in: L'Humanité, 22 décembre 1915.

3 Après la Commune Marx a voulu se débarrasser de la Première Internationale dont il estimait le rôle historique terminé à cause des événements de 1870-71 et, à cause des dissentiments internes, il ne croyait pas le moment propice pour la réorganiser sur un niveau politique. Vaillant, au contraire, pensait que c'était précisément le bon moment pour relancer l'organisation comme l'avant-garde 
légende revolutionnaire de la Commune qui gravite autour de la proscription plus ou moins blanquiste de Londres. ${ }^{1}$ Dans ses écrits de 1872 et de $1874^{2}$ il se livre, ici et là, à des manifestations d' 'impatience révolutionnaire" qui ne manquent pas d'attirer la critique de F. Engels. ${ }^{3}$ Néanmoins il existait toujours une étroite parenté entre ses idées et celles de Marx concernant l'organisation du parti, l'Etat et la Révolution et les leçons de la Commune. Au cours de l'exil ses lettres révèlent deux attitudes parallèles: une analyse lucide et scrupuleusement objective des forces sociales et économiques et des luttes de tendance en France; 4 mais aussi une intransigeance révolutionnaire qui considère toute tentative de redressement socialiste en France tentatives à cette époque forcément très modérées - comme servile, bourgeoise et inutile. ${ }^{5}$

Socialisme marxiste, mais peu doctrinaire, idéalisme révolutionnaire blanquiste: double héritage qui, chez Vaillant se lie en une pensée unique.

La première tâche est, pour lui, l'organisation:

"Aucune révolution plus que celle de la Commune n'a par ses fautes comme par sa grandeur, mieux montré la nécessité de la préparation et de l'organisation. $\|^{6}$

On sait qu'il envisage cette organisation sur les trois plans, politique, économique et démocratique. Bien qu'il poursuive activement ces

politique du prolétariat international. En outre, Marx voulait s'occuper plutôt de l'Allemagne, où les conditions politiques exigeaient une espèce d'alliance avec la bourgeoisie contre l'hégémonie prussienne. Vaillant craignait beaucoup l'influence de cette tactique en France où, après la Commune, une telle alliance était devenue impensable.

1 Au commencement de l'exil l'influence psychologique de la Commune avait été très profonde et Vaillant rêvait d'un "prochain retour offensif de la Révolution" - lettre à J. Ph. Becker du 25 août 1871, Internationaal Instituut voor Sociale Geschiedenis, Fonds Becker, D 59.

2 Internationale et Révolution. A propos du Congrès de la Haye, Londres 1872, brochure expliquant le retrait de l'Internationale des membres vaillantistes; Aux Communeux, Londres 1874, déclaration des réfugiés français. Les deux manifestes, bien que contenant des idées mélangées de marxisme et de blanquisme, témoignent au fond d'une large connaissance des théories marxistes.

3 Engels décèle, cependant, dans le manifeste de 1874 "d'influence incontestable de Vaillant» qui a amené les Français à une adhésion "au programme actuel du communisme allemand."

4 Voir lettre à Andreas Scheu du 27 août 1877, reproduite en annexe (document I).

5 Voir sa réaction au Congrès de Paris de 1876 dans Les Syndicaux et leur Congrès, ainsi que ses lettres à A. Scheu à l'I.I.S.G.

6 E. Vaillant, "Le 18 Mars», in: Républicain socialiste du Centre, 16 mars 1884. 
derniers, et qu'il tire de la Commune des leçons en leur faveur, ${ }^{1}$ ses propres préoccupations et, par conséquent, l'essentiel de l'influence de 1871 se révèlent sur le plan politique.

La première leçon de la Commune, chez Vaillant comme chez Marx, touche le problème de la Révolution et de l'Etat bourgeois. Qu'on n'oublie pas que c'est Vaillant qui, à la Conférence de Londres en 1871 et au Congrès de la Haye de 1872, a proposé la célèbre résolution sur l'action politique. Dans Internationale et Révolution il fait preuve d'une connaissance approfondie de la théorie marxiste concernant ce problème. ${ }^{2}$ A chaque reprise, dans ses articles sur la Commune, il souligne sa vraie valeur politique: elle a montré le chemin en s'emparant du pouvoir; mais elle a échoué en négligeant de briser tout de suite les institutions de l'Etat capitaliste. ${ }^{3}$ Jointe à ceci on trouve chez Vaillant une compréhension profonde de l'importance de la lutte des classes et de la nécessité de bien comprendre les limites admissibles des rapports entre le parti socialiste et l'État bourgeois. Voilà pourquoi, en pleine crise millerandiste, il regrettera que, «de plus en plus reléguée dans l'histoire, la Commune perde peu à peu sa valeur politique». ${ }^{4}$

La deuxième leçon qu'il a tirée de la Commune concerne la nature dynamique, voire dialectique, de l'action. Dans la Commune Vaillant décèle, au fur et à mesure du développement du mouvement, une progression dynamique partant d'un élan patriotique, passant par l'étape d'une lutte entre républicains et monarchistes, aboutissant à l'éclosion d'une conscience socialiste révolutionnaire. ${ }^{\mathbf{5}}$ Cette appréciation des facteurs

1 Ces problèmes sont traités à fond dans la thèse citée plus haut.

2 "Il nous semble que pour des socialistes il n'est pas douteux que, alors qu'auront disparu ces privilèges, ces classes, dont l'existence a produit ce qu'on entend par Etat, Gouvernement, et dont les modifications se manifestent par des changements correspondants de ces institutions, produits de ces distinctions de classes $[\ldots .$.$] et garantie de leur maintien, ces institutions disparaîtront d'elles-$ mêmes, leurs fonctions sociales n'existant plus. [...] Mais pour réaliser cette émancipation des travailleurs, cette abolition des classes [...] il est nécessaire que la bourgeoisie soit dépossédée de son privilège politique, au moyen duquel elle maintient tous les autres. Il faut que dans une période de dictature révolutionnaire le prolétariat emploie pour son affranchissement cette puissance jusqu'ici dirigée contre lui [...] Et seulement alors, quand il aura fait table rase de ces institutions, de ces privilèges qui constituent la société actuelle, cette dictature du prolétariat cessera comme étant sans objet, l'abolition de toute classe faisant disparaître de soi le gouvernement d'une classe." Internationale et Révolution, p. 5. Le texte de cette brochure se trouve dans Etudes de Marxologie, Série S, no 8 [Cahiers de l'I.S.E.A., no 152, août 1964], pp. 163-176.

3 Voir "18 Mars», in: Le Parti Socialiste, 10 mars 1892, et «Vive la Commune», in: La Petite République, 18 mars 1895 . En fait, en mars 1871, Vaillant a conseillé et une attaque immédiate contre Versailles et un assaut sur la propriété. "E. Vaillant, "Anniversaire», in: Le Petit Sou, 15 mars 1901.

- Il tient surtout à saluer le "milieu révolutionnaire et enthousiaste qui [...] 
subjectifs du mouvement révolutionnaire qui, vu les circonstances particulières de la Troisième République, lui permet de remanier le pragmatisme blanquiste d'une façon qui anticipe sur ce que sera la praxis marxiste-léniniste, ${ }^{1}$ l'amène à préconiser "l'action totale» du parti socialiste. ${ }^{2}$

L'aspect le plus important du socialisme ultérieur de Vaillant est peut-être son activité unitaire. Sa formation éclectique et son attitude ouverte ne pourraient que le conduire sur le chemin de l'unité. Mais le souvenir de la Commune y a joué incontestablement un grand rôle aussi. En 1871, Vaillant avait adopté une position nettement unitaire, refusant de se lier avec une fraction quelconque de l'assemblée communale. $^{3}$ Sa conception de l'unité, nationale ou socialiste, fait appel à une association libre de partenaires autonomes - fédéralisme, certes, mais un fédéralisme actif et dynamique. Tout comme Marx, Vaillant a protesté violemment contre l'interprétation qui tendait à faire de la Commune un mouvement décentralisateur, séparatiste. Au contraire, le fédéralisme de Vaillant vise précisément à la création, en commençant par la base, de l'unité de la nation, et ensuite du monde socialiste, tout comme le parti socialiste qu'il envisage naîtra d'un rapprochement loyal des unités déjà existantes, ralliées autour d'un but commun: la réalisation de leur propre unité. Ainsi quand, après le Congrès de Japy, en 1899, les réformistes appelaient à grands efforts à l'existence des fédérations régionales et autonomes, Vaillant, reprenant le mot de Marx, ${ }^{4}$ les traitait de Girondins, et repoussait en même temps leur accusation de Jacobinisme, niant catégoriquement la prédominance, dans son système, d'une ville ou d'un groupement. ${ }^{5}$ Enfin, si les leçons

avait fait, en ces inoubliables et admirables semaines, du peuple de Paris en armes, d'abord pour la garde de ses armes, et contre la réaction et les provocations de Versailles, puis de plus en plus pour l'émancipation ouvrière et la Révolution, un peuple de combattants et de citoyens". La Revue Blanche, mars 1897, p. 72 .

1 Voir J. Howorth, "La propagande socialiste d'Edouard Vaillant», in: Le Mouvement Social, no 72 (septembre 1970), pp. 110-113.

2 Voir interview à Xavier de Ricard, in: Le Parti Socialiste, no 6, 2 août 1891, et son discours du 16 octobre 1908 au Congrès S.F.I.O. de Toulouse, pp. 159-160. Des extraits de ce discours sont reproduits dans l'ouvrage indispensable sur Vaillant: celui de Maurice Dommanget, Paris, La Table Ronde, 1956, pp. 374-76. 3 "Je suis ni de la majorité, ni de la minorité car je n'ai pu trouver un groupe d'hommes avec lequel je puisse marcher." Journal officiel de la Commune du 17 mai. Dans la lettre à Becker déjà citée il déclare que celui avec qui il a "marché le plus d'accord" fut Tridon, blanquiste dissident qui appartint à la "minorité».

4 K. Marx, La Guerre civile en France, Editions sociales 1968, p. 65.

5 «Nos Girondins», in: Le Tocsin populaire du Berry, 24 février 1900. Aussi : «au moment où les invertébrés du socialisme, les fédéralistes retournent spontanément aux formes inférieures de l'organisation, il importe, à l'anniversaire de la 
de la Commune lui dictent la nécessité absolue, à l'heure actuelle, de l'unité socialiste, elles lui montrent aussi que le passé est une source inépuisable de divisions. Ainsi refuse-t-il toujours de remuer les cendres et de mettre en cause soit les personnes soit la littérature de la Commune. ${ }^{1}$

On l'a dit, Vaillant se donna tout entier à l'organisation du socialisme international. Son internationalisme repose sur deux fondements. D'abord, sur le plan idéologique, il souligne toujours la solidarité des peuples, et elle reste symbolisée pour lui par les Frankel, les Dombrowski, ainsi que par la destruction de la colonne Vendôme, symbole de l'esprit de conquête du bonapartisme. ${ }^{2}$ Ensuite, sur le plan pratique, convaincu, dès avant 1871, que la guerre est le moyen le plus efficace dont dispose la bourgeoisie pour détruire la Révolution, et rappelant le premier décret du Comité central sur l'abolition de la conscription, Vaillant dépose, au début de chaque législature, plusieurs propositions visant à une organisation démocratique de l'armée, et surtout à son remplacement par des milices nationales, rappel évident de la Garde nationale de 1871.

Dans ses articles sur la Commune, Vaillant tire, à divers moments, diverses leçons. En 1893, lors de la crise de Panama, il met en lumière l'aspect républicain de la lutte des Communards. En 1893 et en 1896, au moment des Congrès internationaux, c'est l'aspect international qu'il fait ressortir. Au moment de la crise millerandiste, il y montre de préférence la lutte du socialisme révolutionnaire contre la bourgeoisie.

L'autorité qu'a conférée à Vaillant son rôle dans la Commune est immense. En tête du cortège annuel au cimetière du Père-Lachaise, dans les meetings, où sa présence à la tribune suscite spontanément le cri de Vive la Commune!, il est devenu le symbole vivant de la lutte de Paris. A cet égard on pourrait peut-être conclure en constatant que la légende de la Commune est arrivée, bon gré, mal gré, à mettre dans l'ombre le fonds d'originalité du socialisme moderne, pourtant constamment prêché par Vaillant: l'action totale du parti socialiste.

Commune que [...] nous nous rendions compte de la nécessité d'achever notre œuvre de concentration. [L']unité, c'est la vraie revanche de la Commune." "Commune et Unité", in: Le Petit Sou, 13 mars 1902.

1 Kautsky lui ayant demandé d'écrire un article pour le Vorwärts qui évaluerait les divers ouvrages jusqu'alors consacrés à la Commune, Vaillant lui répond: «je me suis toujours gardé d'articles critiques des livres qui prétendaient être l'histoire de ses hommes et de ses événements». Un tel article l'obligerait de traiter les "questions de personnes" et ceci est le "motif principal" de son refus. Lettre à Kautsky du 6 novembre 1905, I.I.S.G., Fonds Kautsky, D XXII 339. 2 Voir "Guerre et Commune», in: Le Socialiste, 13 mars 1904. Cf. Marx: La Guerre civile, op. cit., pp. 71-72 et 92 . 


\title{
DOCUMENTS
}

\section{LETTRE DE VAILLANT A ANDREAS SCHEU DU 27 AOUT 1877}

\begin{abstract}
Vaillant avait fait la connaissance d'Andreas Scheu en 1868-69 lorsque les deux hommes étaient étudiants à Vienne. Il entretenait avec lui une vive correspondance qui ne cessait qu'avec sa mort en 1915. Ces lettres sont conservées a l'Internationaal Instituut voor Sociale Geschiedenis, Amsterdam (fonds A. Scheu).

La lettre reproduite pour l'essentiel ci-dessous est d'une netteté admirable pour établir l'état d'évolution des idées politiques de Vaillant en 1877 et doit être considérée comme un complément indispensable aux manifestes: Internationale et Révolution (1872), Aux Communeux (1874) et Les Syndicaux et leur Congrès (1876).
\end{abstract}

J'ai reçu les 2 nos des "Droits de l'homme" et désire que vous y ayez trouvé les éléments utiles pour avertir au besoin vos amis de la valeur des déclamations des Buffenoir et Cie. ${ }^{1}$ Toutes les fois que je verrai quelque chose qui puisse vous éclairer sur la situation vraie des choses en France ou qui puisse corriger quelqu'erreur de vous ou de vos amis je vous l'enverrai. De même je vous dirai volontiers tout ce qui peut vous aider à voir cette situation et à savoir ce qu'en pense le parti révolutionnaire. Nous sommes bien loin d'être indifférents à ce que pense le peuple allemand des choses de la Révolution en France, et vous auriez tort de le croire. Si à toute occasion je vous parle de ces choses c'est, autant que par affection pour vous, pour que vous sachiez - et cela aussi bien pour vos amis que pour vous - notre pensée à ce sujet. Nous n'hésiterions donc pas a faire connaître directement cette pensée, si nous avions moyen de le faire. Dans ma dernière lettre je vous disais déjà à quel point nous apprécions ce moyen de propagande générale qui s'adresse à la masse, qui est le levier de l'agitation: la publicité et surtout par le journal. Je vous disais aussi quelles difficultés il y avait pour nous et comment nous n'y pouvions penser qu'en trouvant ou créant les conditions de réalisation. Il va sans dire que seulement alors nous pourrions directement parler à l'étranger ou plutôt mettre nos amis de l'étranger à même de connaître nos idées et notre politique, comme le "Vorwärts" rend possible aux Français de connaître les idées et la politique du parti socialiste officiel en Allemagne. ${ }^{2}$ En attendant, hors des cas exceptionnels, nous ne pouvons nous adresser au public d'une façon utile. Ces cas exceptionnels sont ceux où il s'agit d'affirmer l'idée révolutionnaire après la défaite ou la dégager d'entreprises suspectes. C'est ce qui a produit les deux brochures de: «aux communeux" et des "syndicaux". Dans la crise officielle actuelle des partis lé-

1 Hippolyte Buffenoir, rédacteur aux Droits de l'Homme, radicalisant, ex-ami de Gambetta, membre du groupe du café Soufflot qu'animera plus tard Jules Guesde. Se mêla à la campagne d'Emile Acollas en 1876 et, étroitement lié au parti légaliste allemand, se trouve souvent visé par les dards de Vaillant qui exécrait à cette époque l'avilissement exigé par la participation plus ou moins futile aux jeux électoraux.

2 Internationaliste au plein sans du terme, Vaillant souligne toujours la nécessité d'ouvrir des moyens de communication entre les partis socialistes des divers pays. 
gaux en France ${ }^{1}$ la politique révolutionnaire est trop nette, notre idée a été trop nettement exprimée pour qu'aucun doute soit possible. Cette crise n'est que le conflit arrivé à l'état aigu de ces partis versaillais de droite et de gauche qui se disputent le pouvoir depuis la chute de la Commune. En attendant le recours possible du pouvoir actuel - en cas de défaite - à des moyens plus violents, le débat est porté sur le terrain électoral. Il s'agit donc pour la masse électorale de savoir si elle va se prononcer pour la République bourgeoise ou pour la monarchie cléricale. Nous ne sommes pas indifférents au résultat, nous verrions dans le triomphe électoral de la droite une déchéance, une défaillance du pays, un abandon et une lâcheté semblable à celle qui suivit le 2 Décembre 1851, ce serait la France morte à la Révolution pour des générations et une chute nationale à laquelle nous ne pouvons non plus être indifférents, car elle ferait disparaître les conditions mêmes de production et maintien de la Révolution en notre pays. La victoire de la gauche serait le maintien de l'état actuel avec cette différence cependant que l'agitation de la crise actuelle ne permettrait pas à ces intrigants de gauche de faire rentrer la masse nationale dans le sommeil des temps précédents. L'illusion populaire qui les aurait fait triompher amènerait comme conséquences des résultats qu'ils détestent, mais auxquels ils seraient peu à peu entraînés malgré eux. Ce serait loin d'être une victoire populaire mais ce serait le commencement de la lutte décisive entre le peuple et les dernières troupes de la bourgeoisie. ${ }^{2}$ Ce serait une preuve que la terreur versaillaise a perdu son pouvoir, que le prêtre n'effraye plus, qu'il perd son pouvoir, est aussi méprisé que haï et qu'enfin la lâcheté de la masse populaire ne lui fait pas abandonner les dernières des conquêtes de la 1 ere révolution. Ce serait aussi les probabilités d'une guerre

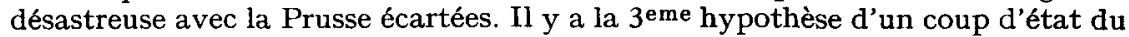
pouvoir de droite réduit à un coup de force en cas de défaite électorale. Coup d'état fort désiré par la fraction bonapartiste surtout mais que les divisions et rivalités des fractions de droite rendent peu probable et en cas de tentative, de succès douteux. Cette tentative amènerait alors entre les partis versaillais une lutte plus active que la lutte électorale, l'armée déciderait. Dans les trois cas les vainqueurs auraient toujours pour ennemi principal non le vaincu du jour qui sous une autre forme deviendrait le complice du lendemain; mais le vaincu, l'opprimé des gauches et droites victorieuses, serait toujours le vaincu de la commune, le peuple maintenu sous l'oppression et l'exploitation de la monarchie cléricale, du brigandage bonapartiste ou de la République bourgeoise. Si donc dans le parti que prendra la masse nationale nous avons un sentiment fort loin de l'indifférence, c'est que nous voyons dans ce parti pris par cette masse l'indication du degré de vitalité de cette même masse qui est la matière même, la source de la vie nationale, le milieu d'où sortent et qui forme les manifestations de cette vie, les reculs ou la marche vers la Révolution, parce que suivant l'état de cette masse nationale elle est plus ou moins mobile vers la révolution, plus ou moins prête à subir l'influence révolutionnaire des événements et l'impulsion, la loi du parti révolutionnaire. ${ }^{3}$ Mais cette impulsion, cette loi du parti révolu-

1 Référence à la crise du 16 Mai 1877.

2 Analyse tout à fait marxiste du mouvement historique et dialectique de la lutte des classes.

3 Ici l'on remarquera la méthode révolutionnaire qui, alliant l'étude objective des forces sociales, économiques et politiques à la préparation subjective de la masse populaire, autrement dit de la conscience socialiste révolutionnaire du prolétariat, préconise la mise en marche de la praxis marxiste-léniniste. 
tionnaire, seront d'autant plus fortes que le parti révolutionnaire se sera plus fortement constitué et n'aura à aucun moment de la crise ou de la série de crises entre les partis versaillais pactisé avec aucun d'eux. ${ }^{1}$ Les communeux ennemis du suffrage ne peuvent pas plus entrer dans la lutte électorale de leurs deux ennemis de gauche et droite qu'ils ne pourraient en cas de lutte armée prendre les armes pour aucun côté. Ils ne sont pas assez forts en ce moment pour combattre leur propre combat à eux seuls, le combat de la Révolution. Ils doivent donc attendre dans la propagande pour leur idée et l'organisation de leurs forces le moment où ils pourront commencer ce combat. En attendant ils suivront avec intérêt les péripéties du combat que se livrent leur adversaires, profitant pour la Révolution de tous les incidents, suivant avec intérêt et même passion toutes les manifestations de la vie nationale qui se produiront; mais ne cédant à aucune des sollicitations des charlatans qui voudraient les entraîner à des compromis, fussent-ils d'un jour, avec les éléments soit disant [sic] "intransigeants" de Versailles, non moins détestables et bourgeois qu'ils se nomment Louis Blanc, Naquet ou Buffenoir que s'ils étaient Thiers ou Gambetta. En dehors de ce qui précède il y a évidemment pour l'avenir de la Révolution intérêt, comme vous l'avez très bien jugé, à ce que la lutte entre le peuple et la bourgeoisie se produise sous la forme républicaine. Le parti révolutionnaire a toujours intérêt à la netteté des situations et il n'y a pas de situation plus nette que celle qui mettrait en opposition le parti révolutionnaire, le peuple, et la bourgeoisie radicale atteignant avec les Louis Blanc etc. la limite des concessions, puis maintenant avec la même fureur que la monarchie la distinction de classes, l'exploitation et la répression populaire. Comme vous le dites très bien, dans la lutte contre une monarchie ou un empire les républicains versaillais hypocritement mêlés au peuple feraient oublier leurs crimes passés, leur situation réelle d'hommes de la bourgeoisie, et créeraient un gâchis des ténèbres que tous les efforts des révolutionnaires ne pourraient éclairer. Par la forme monarchique le peuple se trouverait rejeté dans la politique confuse, dans le mysticisme politique du passé. Dans la forme républicaine, c'est-à-dire dans le gouvernement direct de la bourgeoisie sans intermédiaire royal ou impérial, le gouvernement d'une classe maîtresse finirait par se montrer clairement aux plus aveugles, et l'abolition des classes par la Révolution se montrerait avec la même évidence comme la seule issue et pressante de la société d'oppression actuelle. Tous les partis intermédiaires et modérés devraient bientôt disparaître et il ne resterait plus en présence que bourgeoisie et prolétariat, réaction et révolution. La seule objection qu'on puisse faire est que dans les crises que provoque certainement l'établissement d'une monarchie dans un pays aussi divisé dans sa bourgeoisie (par tant d'appétits) que l'est la France, il y a lieu et occasion à ces jours de crise pour le parti révolutionnaire de saisir ces occasions et d'entraîner le pays à la Révolution. On ne peut nier que ces chances peuvent en effet se produire; mais elles sont difficiles à saisir, les circonstances peu favorables, et le trouble de la situation amène presque toujours l'opposition quant à la forme et la continuation en réalité du pouvoir qui vient d'être renversé, ainsi qu'il est arrivé en Février 1848 et au 4 Septembre 1870 et, après un intermède révolutionnaire de quelques jours, l'aile gauche de l'armée bourgeoise est venue relayer l'aile droite. Il est certain qu'il est du devoir du parti révolutionnaire de s'organiser et de se préparer de façon que ces intermèdes du passé deviennent une situation définitive

1 Attitude qui renaîtra en 1888 pendant la crise boulangiste et aussi en 1899 lors de la constitution du ministère Waldeck-Rousseau/Millerand/Galliffet. 
et que la crise devienne une révolution. Mais il est préférable, quand même l'assaut du pouvoir devrait être plus long en apparence, quand même la lutte du prolétariat contre la bourgeoisie semblerait voir diminuer des chances accidentelles, il est préférable que cet assaut soit le dernier, que cette lutte soit définitive, qu'il n'y ait plus de place pour les partis moyens et que la chute du pouvoir bourgeois soit équivalente avec l'anéantissement de la bourgeoisie, l'abolition des classes, la conquête de la société égalitaire nouvelle. ${ }^{1} \mathrm{Je}$ vous ai parlé aussi longtemps de ces choses quoique je n'aie fait que vous répéter une masse de choses que vous savez aussi bien que moi, je vous en ai parlé aussi longtemps seulement pour vous bien indiquer ce que je pense de la situation en France et combien tout en approuvant l'attaque du "Vorwärts» contre l'extrême gauche nous différons sur sa façon d'envisager "l'extrême gauche», la presse radicale etc., qu'ils ne condamnent qu'autant qu'ils s'unissent au reste des gauches. Ce qui veut dire qu'entraînée par sa situation légale anti-révolutionnaire, la tête du parti officiel socialiste en Allemagne fait la guerre indirectement (comme l'Internationale) aux révolutionnaires français pour chercher des alliés aux confins de Versailles.

\section{FEVRIER 1892: DECLARATION DU COMITE REVOLUTIONNAIRE CENTRAL}

Après la scission du mouvement blanquiste en 1889, Vaillant se trouve enfin libre pour orienter son parti dans le sens ouvert et actif qu'il préconise depuis longtemps. Il ne tarde donc pas à publier une déclaration et règlement. Le document qui suit, la "déclaration", date de janvier 1892. Bien que totalement inédit, sauf dans les publications du C.R.C. datant de l'époque, ${ }^{2}$ il devrait être considéré comme l'expression la plus nette de la politique du C.R.C. épuré et vénové - le parti vaillantiste.

\section{DECLARATION}

Convaincu que dans le mouvement socialiste international, le rôle des nations est, indépendamment des causes générales et communes, déterminé pour chacune, par sa nature, son histoire et les circonstances, le C.R.C. s'est toujours proposé de marcher dans la voie de la tradition révolutionnaire du peuple français, pour qui toute revendication sociale, dès qu'elle se généralise et devient plus active, prend un caractère essentiellement politique. ${ }^{3}$

1 Ainsi Vaillant non seulement refuse-t-il d'envisager la tactique associée étroitement avec l'activité blanquiste du Second Empire, mais il choisit même, consciemment, de lutter sur un nouveau terrain avec de toutes autres méthodes. ${ }^{2}$ Le texte complet a été publié dans chaque numéro du Parti Socialiste à partir du 21 février 1892 (no 35, p. 1). Nous ne donnons ici que la Déclaration.

${ }^{3}$ Il faut se garder de déceler, dans les références à la "tradition révolutionnaire française" et aux traits nationaux caractéristiques, la prédominance de préoccupations d'ordre "blanquiste». En effet, Vaillant ne fait que constater l'existence de ces traits nationaux, et ne s'occupe point de la question de leurs origines. Mais, étant donné leur existence, il faut choisir spécialement les moyens de lutte pour répondre à leurs besoins; autrement dit, il faut adapter le marxisme aux exigences d'une situation française. C'est ici que Vaillant se sépare des doctrinaires du P.O.F. 
Le C.R.C. et tout socialiste digne de ce nom reconnaît la lutte de la classe ouvrière contre la classe capitaliste, comme la caractéristique du socialisme actuel, la règle directrice de son action: pour l'émancipation de la classe ouvrière, pour le combat, la victoire de la Révolution contre la réaction, pour la délivrance enfin de tous les jougs politiques, sociaux et intellectuels de l'homme, dans la liberté individuelle et collective, dans l'égalité de fait et la solidarité de la République sociale, de la société sans Dieu ni maître. Il est athée (matérialiste et transformiste ${ }^{1}$ ), républicain, communiste, révolutionnaire et internationaliste.

En dehors, cependant, de l'action consciente qui, aux lenteurs hasardeuses d'une évolution historiquement déterminée, inévitable, mais d'une allure incertaine, substitue le cours rapide de la Révolution, chaque jour par la force des choses, par l'effet de sa puissance infiniment accrue de production, d'intelligence et d'énergie, la société brise les formes mortes, rompt les liens qui l'enserrent et prépare, forme l'avenir.

Il n'est pas un de ces effets, de ces résultats, si minime soit-il, qui ne soit désirable. Il n'est paș un des actes de la vie nationale auquel nous ne devions participer, dont nous ne devions tenir compte et tirer parti dans l'intérêt des revendications ouvrières et pour le développement de la République.

Si, entre toutes réformes, nous mettons avant tout, celles qui, plus directement, importent à notre cause: les conditions et garanties du travail, l'organisation et la liberté syndicale, assurées par une législation nationale et internationale; il n'est aucun progrès des institutions républicaines, des droits municipaux, des libertés individuelles et populaires, de l'instruction publique élémentaire et supérieure unifiée et généralisée, de l'hygiène, des arts, des sciences, de l'industrie et de tout mode de l'activité humaine, qui nous soit indifférent. Ce sont autant de pas hors du passé, autant de moyens qui, bientôt, socialement employés, permettront de répandre sur tous les biens, les richesses, l'aisance, le bonheur, aujourd'hui monopolisés.

Le C.R.C. considère la paix internationale comme la condition essentielle et première de cette éclosion progressive et irrésistible de la société nouvelle; aussi le militarisme et le césarisme sont-ils ses plus directs ennemis; et il réclame tout d'abord: la suppression de l'armée permanente et l'armement général du peuple, militairement instruit et organisé, pour la défense de son indépendance et la garde de ses droits.

Au lieu d'attendre dans l'impuissance de divisions, auxquelles nous avons toujours été étrangers, la débâcle d'un état de choses anarchique, que maintient à grand'peine le privilège propriétaire et capitaliste, armé du pouvoir, nous voulons agir sans retard et constituer dans son unité d'action, préparant son unité d'organisation, le parti socialiste, l'avant-garde de l'armée ouvrière ralliée, entraînée, menée à l'assaut de la société capitaliste, à la conquête du pouvoir politique.

C'est au premier rang de cette avant-garde militante que nous voulons, pour combattre, désarmer la réaction pour armer la Révolution, de cet instrument tout puissant - le pouvoir - qui, aux mains de l'ennemi, fait notre misère et notre faiblesse, et qui demain fera notre force et notre délivrance.

1 Le seul fait de se réclamer de l'athéisme a pu suffire à classer Vaillant dans le camp blanquiste. Mais comme l'on voit, après ces qualificatifs, Vaillant aborde la question de l'athéisme dans un esprit plus dialectique et "scientifique» que ne l'abordait Blanqui pour qui l'athéisme était vraiment la base philosophique de sa critique sociale. 
Maîtres du pouvoir politique, le parti socialiste et la classe ouvrière arracheront à la classe possédante, au privilège, aux accapareurs de la société capitaliste, la matière et les instruments de production, rendus propriété sociale, fonctionnant pour la communauté sociale, dans l'ordre moral de la société socialiste pouvant alors évoluer librement. - A cette conquête émancipatrice du pouvoir politique, à l'avènement de cette dictature impersonnelle et initiatrice de l'ordre nouveau, du prolétariat socialiste, nous devons employer tous les moyens, donner tous nos efforts.

Le programme général du C.R.C. est celui que dans le Congrès de l'Internationale, interrompu par les massacres versaillais, puis dans les Congrès internationaux de Paris (1889), de Bruxelles (1891) et dans les suivants ont formulé et formuleront les délégués de la classe ouvrière et du parti socialiste, le programme vivant du prolétariat socialiste, arrêté par lui, toujours développable, en rapport avec les progrès des idées, des mcurs, des événements et des choses.*

Son principe d'action socialiste et révolutionnaire, c'est: éclairé par les lumières du socialisme moderne, en dehors de toute limitation doctrinaire ou sectaire, de combattre incessamment, à toute occasion, sur tous les terrains, le combat de la classe ouvrière, du socialisme et de la Révolution, jusqu'à l'abolition du régime capitaliste, des privilèges et des classes.

Parti d'action, le C.R.C. adopte tous les modes d'activité économique, politique et sociale, électorale et révolutionnaire.

Il ne s'interdit d'autres actes que ceux qui, même seulement en apparence, contredisant au programme et à l'idée socialiste ou à l'honneur, ravaleraient l'action socialiste au niveau des combinaisons politiciennes des partis bourgeois, la discréditeraient et déshonoreraient. ${ }^{1}$ Il n'est pas deux morales, l'une privée et l'autre publique, comme l'ont dit ou pratiqué tous les politiciens. La loyauté est le devoir rigoureux, la seule vraie et habile tactique du socialisme.

Le C.R.C., inébranlablement fidèle à la fois à la République, au Socialisme et à la Révolution, insensible aux attaques, dédaigneux de toute intrigue, châtiant les trahisons, a su, dans les crises dernières, combattre le césarisme sans fléchir devant l'opportunisme. Si son appel eût été mieux écouté, le boulangisme eût été écrasé sans que l'opportunisme eût pu triompher. Le C.R.C. a toujours combattu et combattra toujours la réaction, sous quelque forme du

* Les Congrès de l'Internationale et les Congrès internationaux socialistes ont voté et nous affirmons avec eux: "La manifestation du 1 er Mai ; le pacte international d'union des travailleurs et des socialistes de tous les pays; la lutte de classe; la journée de huit heures; la salaire minimum équivalent aux besoins; la législation nationale et internationale protectrice du travail; le combat par tous les moyens contre le militarisme et la guerre, pour la suppression de l'armée permanente, pour la paix internationale; l'abolition du salariat et du patronat, du privilège et du régime capitaliste par la socialisation de la matière et des instruments de production, etc. etc.; enfin l'organisation nationale et internationale de la classe ouvrière et du parti socialiste; le secrétariat du travail et la périodicité des Congrès."

Le C.R.C. a ses représentants aux Congrès internationaux socialistes et au secrétariat du travail.

1 A cette époque Vaillant ne laissait passer aucune occasion de critiquer ses anciens camarades passés dans le camp rochefortiste. 
masque qu'elle se présente ou déguise: partis du passé ou du présent; intrigues césarienne ou monarchique; religieuse et cléricale; contre-révolution opportuniste, administrative et gouvernementale.

Dans de nombreuses déclaratıons, dont celle-ci est le résumé, dans les propositions de ses élus, le C.R.C. a marqué le caractère de son programme spécial, et il ne cessera d'affirmer nettement par ses actes et ses paroles, en toute occasion, ses opinions et volontés, son action, son rôle, dans le mouvement socialisterévolutionnaire. 